\title{
Pre-hibernation performances of the OSIRIS cameras onboard the Rosetta spacecraft
}

\author{
S. Magrin ${ }^{1,2}$, F. La Forgia ${ }^{2}$, V. Da Deppo ${ }^{3}$, M. Lazzarin ${ }^{2}$, I. Bertini ${ }^{1}$, F. Ferri ${ }^{1}$, M. Pajola ${ }^{1}$, M. Barbieri ${ }^{1}$, G. Naletto ${ }^{1,4}$, \\ C. Barbieri ${ }^{1,2}$, C. Tubiana ${ }^{5}$, M. Küppers ${ }^{6}$, S. Fornasier ${ }^{7,8}$, L. Jorda ${ }^{9}$, and H. Sierks ${ }^{5}$ \\ ${ }^{1}$ Centro di Ateneo di Studi e Attivitá Spaziali “Giuseppe Colombo", University of Padova, via Venezia 15, 35131 Padova, Italy \\ e-mail: sara.magrin.1@unipd.it \\ ${ }^{2}$ Department of Physics and Astronomy, University of Padova, vicolo dell'Osservatorio 3, 35122 Padova, Italy \\ 3 CNR-IFN UOS Padova LUXOR, via Trasea 7, 35131 Padova, Italy \\ ${ }^{4}$ Department of Information Engineering, University of Padova, via Gradenigo 6, 35131 Padova, Italy \\ 5 Max-Planck-Institut für Sonnensystemforschung, Justus-von-Liebig-Weg 3, 37077 Göttingen, Germany \\ ${ }^{6}$ European Space Astronomy Centre, ESA, Villanueva de la Cañada, 28691 Madrid, Spain \\ 7 LESIA, Observatoire de Paris, CNRS, UPMC Univ Paris 06, Univ. Paris Diderot, 5 Place J. Janssen, 92195 Meudon Pricipal Cedex, \\ France \\ ${ }^{8}$ Université Paris Diderot, Sorbonne Paris Cité, 4 rue Elsa Morante, 75205 Paris, France \\ 9 Laboratoire d'Astrophysique de Marseille, CNRS and Université de Provence, 38 rue Frédéric Joliot-Curie, 13388 Marseille, France
}

Received 18 March 2014 / Accepted 6 December 2014

\section{ABSTRACT}

\begin{abstract}
Context. The ESA cometary mission Rosetta was launched in 2004. In the past years and until the spacecraft hibernation in June 2011, the two cameras of the OSIRIS imaging system (Narrow Angle and Wide Angle Camera, NAC and WAC) observed many different sources. On 20 January 2014 the spacecraft successfully exited hibernation to start observing the primary scientific target of the mission, comet 67P/Churyumov-Gerasimenko.

Aims. A study of the past performances of the cameras is now mandatory to be able to determine whether the system has been stable through the time and to derive, if necessary, additional analysis methods for the future precise calibration of the cometary data.

Methods. The instrumental responses and filter passbands were used to estimate the efficiency of the system. A comparison with acquired images of specific calibration stars was made, and a refined photometric calibration was computed, both for the absolute flux and for the reflectivity of small bodies of the solar system.

Results. We found a stability of the instrumental performances within $\pm 1.5 \%$ from 2007 to 2010 , with no evidence of an aging effect on the optics or detectors. The efficiency of the instrumentation is found to be as expected in the visible range, but lower than expected in the UV and IR range. A photometric calibration implementation was discussed for the two cameras.

Conclusions. The calibration derived from pre-hibernation phases of the mission will be checked as soon as possible after the awakening of OSIRIS and will be continuously monitored until the end of the mission in December 2015. A list of additional calibration sources has been determined that are to be observed during the forthcoming phases of the mission to ensure a better coverage across the wavelength range of the cameras and to study the possible dust contamination of the optics.
\end{abstract}

Key words. instrumentation: detectors - space vehicles: instruments - techniques: photometric

\section{Introduction}

The ESA Rosetta mission was launched on 2 March 2004, and since then, the Optical, Spectroscopic, and Infrared Remote Imaging System (OSIRIS, Keller et al. 2007), which is a twocamera system, performed many different scientific observations. It acquired data during a swing-by with Mars and Phobos in 2007, a fly-by with asteroid (2867) Steins in 2008, two swingbys with Earth in 2007 and 2009, and a fly-by with asteroid (21) Lutetia in 2010, to mention only the main activities. On 8 June 2011, the spacecraft entered into deep-space hibernation mode. It successfully exited hibernation on 20 January 2014 for the cruise to the rendez-vous with the primary scientific target of the mission: the Jupiter-family comet $67 \mathrm{P} /$ ChuryumovGerasimenko.

Establishing the stability of the instrument through time is vital to performing a correct absolute flux calibration of the cometary data.
In Sect. 2 we therefore study the past performances of the two cameras, the Narrow Angle and Wide Angle Camera (NAC and WAC), through the observations of calibration stars. In particular, we investigate the past responses of calibrator signal through time with different filters to be able to evaluate possible aging effects on the optics and to determine whether the photometric requirements set by the original mission science goals have remained stable. We also inspect the dynamical range of the two CCDs by checking the linear dependence between the stellar signal and the exposure time of observations. The transmission and reflectivity curves of each component of the two cameras, measured during on-ground calibration tests, is used to estimate the efficiency of the instrumentation. We thus check and compare the expected calibrator signals that are deduced from these instrumental efficiency curves and from the observed one, which is computed from images acquired in flight.

Following this investigation, we present in Sect. 3 the robust formulation for the absolute flux calibration of OSIRIS images. 
Table 1. Basic parameters of the NAC and WAC system and CCD specification (Keller et al. 2007).

\begin{tabular}{|c|c|c|}
\hline & NAC & WAC \\
\hline Optical design & 3-mirror off-axis & 2-mirror off-axis \\
\hline Detector type & $2 \mathrm{k} \times 2 \mathrm{k} C \mathrm{CD}$ & $2 \mathrm{k} \times 2 \mathrm{k} C \mathrm{CD}$ \\
\hline Angular resolution $\left(\mu \mathrm{rad} \mathrm{px}^{-1}\right)$ & 18.6 & 101 \\
\hline Focal length (mm) & 717.4 & $140(\mathrm{sag}) / 131(\tan )$ \\
\hline Field of view $\left(^{\circ}\right)$ & $2.20 \times 2.22$ & $11.35 \times 12.11$ \\
\hline Telescope aperture & $6.49 \times 10^{-3} \mathrm{~m}^{2}$ & $4.91 \times 10^{-4} \mathrm{~m}^{2}$ \\
\hline F-number & 8 & 5.6 \\
\hline Spatial scale from $1 \mathrm{~km}\left(\mathrm{~cm} \mathrm{px}^{-1}\right)$ & 1.86 & 10.1 \\
\hline & \multicolumn{2}{|c|}{ NAC and WAC CCD } \\
\hline Pixel size & \multicolumn{2}{|l|}{$13.5 \times 13.5 \mu \mathrm{m}^{2}$} \\
\hline Full well & \multicolumn{2}{|l|}{$>120000 \mathrm{e}^{-} \mathrm{px}^{-1}$} \\
\hline System gain & \multicolumn{2}{|l|}{$\approx 3.1 \mathrm{e}^{-} / \mathrm{DU}$} \\
\hline Readout noise (CCD) & \multicolumn{2}{|l|}{$\approx 15 \mathrm{e}^{-} \mathrm{rms}$} \\
\hline Dark charge generation & \multicolumn{2}{|c|}{$\begin{array}{l}<0.1 \mathrm{e}^{-} \mathrm{s}^{-1} \mathrm{px}^{-1} @ 180 \mathrm{~K} \\
\approx 400 \mathrm{e}^{-} \mathrm{s}^{-1} \mathrm{px}^{-1} @ 293 \mathrm{~K}-\text { (with dithering) }\end{array}$} \\
\hline Readout rate & \multicolumn{2}{|c|}{ 1.3 $\mathrm{Mpx} \mathrm{s}^{-1} ; 650 \mathrm{kpx} \mathrm{s}^{-1}$ per channel } \\
\hline Readout time (full frame) & \multicolumn{2}{|c|}{$3.4 \mathrm{~s}$ (2 channels) } \\
\hline
\end{tabular}

Table 2. NAC and WAC filter names and wheel positions.

\begin{tabular}{lrlrl}
\hline \hline & \multicolumn{2}{c}{ NAC } & \multicolumn{2}{c}{ WAC } \\
Position & Wheel 1 & Wheel 2 & Wheel 1 & Wheel 2 \\
\hline 1 & FFP_UV & FFP_IR & empty & empty \\
2 & FFP_Vis & Orange & Green & Red \\
3 & NFP_Vis & Green & UV 245 & UV 375 \\
4 & Near-IR & Blue & CS & CN \\
5 & Ortho & Far-UV & UV 295 & NH2 \\
6 & Fe2O3 & Near-UV & OH & Na \\
7 & IR & Hydra & UV 325 & OI \\
8 & Neutral & Red & NH & Vis 610 \\
\hline
\end{tabular}

Notes. Position of the two filter wheels of all filters for NAC and WAC. In principle, every combination is possible, but only some of them are useful for scientific purposes.

Section 4 is dedicated to the description of the conversion between the signal observed on the surface of solar system bodies and of their correct geometric reflectance.

The NAC is a three-mirror anastigmat system, while the WAC has an off-axis optical configuration obtained with two aspherical mirrors (Keller et al. 2007). In Table 1 we report the main characteristics of the NAC and WAC system, together with the specifications of the CCDs.

A plane-parallel antireflection coated plate, referred to as antiradiation plate (ARP), was added in front of the CCD for radiation shielding.

Both cameras are equipped with two filter wheels placed in front of the CCD. A passband is defined by a combination of two filters, hereafter $(x, y)$, where $x$ is the filter position on the first wheel and $y$ is the position on the second wheel. In Table 2 we list all filters available for the two cameras by specifying their position on the wheels.

The WAC filters are located in seven of the eight positions of the two filter wheels, from 2 to 8 , leaving the first position in both wheels empty. All seven filters of the first wheel can be used by setting the empty position in the second one, and vice-versa.

In the NAC the filter wheels have no empty position. Neutral or clear filters are used in combination with band-pass filters to optimize the camera performances either in the UV, in the visible, or in the IR range. A different focus range for near focus plate - visible (NFP-Vis) with respect to far focus plate - visible (FFP-Vis) is also planned, which will allow optimizing the resolution of the NAC for observations of the comet surface at a distance shorter than $4 \mathrm{~km}$.

We can therefore define the filters as twins, which can be obtained by combining different clear filters in addition to the same band-pass filter. These combinations share the same passband, but have different efficiencies in general because clear filters have different efficiencies. Together with defining twin combinations, it is also possible to define a baseline filter combination by selecting a representative of the group and deduce the flux calibration of the whole group according to the baseline calibration.

In Appendix A we list and define the physical parameters and units we used.

\section{Performance of OSIRIS cameras}

\subsection{Expected count rate}

The expected total count rate, $K_{x, y}$ in $\mathrm{DN} \mathrm{s}^{-1}$, of a given source on an image acquired through the $(x, y)$ filter combination is expressed by

$$
\begin{gathered}
K_{x, y}=\int_{0}^{\infty} \varphi(\lambda) \cdot M^{n}(\lambda) \cdot F_{x, y}(\lambda) \cdot T_{\mathrm{ARP}}(\lambda) \\
\quad \times Q(\lambda) \cdot \frac{\lambda}{h c} \cdot \frac{1}{\mathrm{IG}} \cdot \operatorname{AP} \mathrm{d} \lambda
\end{gathered}
$$

where

$-\varphi(\lambda)$ is the spectral irradiance of the source at the camera entrance aperture, expressed in $\mathrm{W} \mathrm{m}^{-2} \mathrm{~nm}^{-1}$;

- $Q(\lambda)$ is the CCD quantum efficiency expressed in $\mathrm{e}^{-} / \mathrm{ph}$;

- IG is the inverse gain, which hereafter is taken to be $3.1 \mathrm{e}^{-} / \mathrm{DN}$;

- $M^{n}(\lambda), F_{x, y}(\lambda), T_{\mathrm{ARP}}(\lambda)$ are the mirror spectral reflectance (through $n$ mirrors: $n=2$ for WAC or $n=3$ for NAC), filters, and antiradiation plate (ARP) transmissions as a function of the wavelength;

- $A P$ is the telescope aperture: $4.91 \times 10^{-4} \mathrm{~m}^{2}$ for the WAC and $6.49 \times 10^{-3} \mathrm{~m}^{2}$ for the NAC.

In Fig. 1 we report the reflectivity of the three-mirror (NAC) and two-mirror (WAC) systems together with filters and ARP transmission curves. 

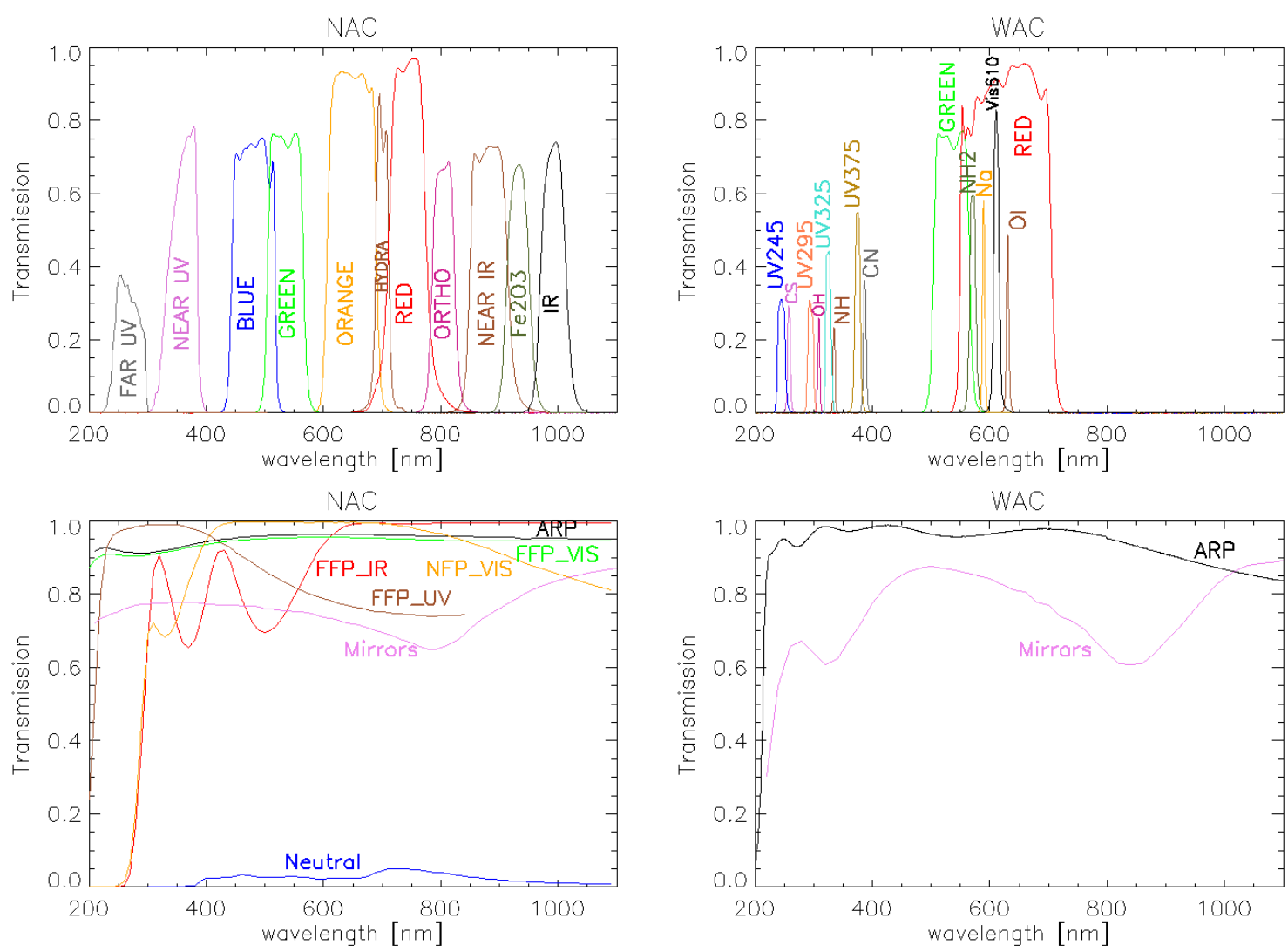

Fig. 1. Efficiency curves of NAC and WAC optics: transmission curves of clear and band-pass filters, antiradiation plates (ARP), and reflectivity of mirrors.

The integrand of Eq. (1) is the wavelength dependance of the count rate detected by the instrument. In particular, $Q(\lambda) \times$ $\lambda / h c$ is the way to express the CCD efficiency in $\mathrm{e}^{-} /(\mathrm{W} \mathrm{s})$, where $\lambda /(h c)=\lambda /\left(1.986 \times 10^{-16}\right)$ if $\lambda$ is expressed in $\mathrm{nm} . Q(\lambda) \times \lambda /(h c$. $\mathrm{IG})$ hence gives the CCD efficiency in $\mathrm{DN} /(\mathrm{W} \mathrm{s})$.

If the source flux is constant with time, then $\varphi(\lambda)$ is time independent. Since in principle all instrument optics responses depend on age, the integrand of Eq. (1) and hence $K_{x, y}$ are therefore time (age) dependent.

\subsection{Observed count rate}

Equation (1) gives the total count rate that is expected on the CCD in each $(x, y)$ passband of a source with a known spectral irradiance $\varphi(\lambda)$. As a first check of the OSIRIS performances, we compared these theoretical values with the real values observed by the instrument.

Two stars were regularly observed by OSIRIS during the past mission phases for calibration purposes: Vega and the double star 16 Cyg A+B. 16 Cyg B is a solar analog and is suitable for measuring the reflectance of small bodies of the solar system. The corresponding spectral irradiances that were taken into account to provide the theoretical values we compared our values with were taken from Bohlin \& Gilliland (2004) for Vega and from Burlov-Vasiljev et al. $(1995,1998)$ for the solar spectrum properly scaled to match $16 \mathrm{Cyg} \mathrm{A}$ and B.

\subsubsection{Aperture photometry}

We derived the observed count rate from the calibration stars with the aperture photometry method.
The raw data were processed to remove the bias level. Flatfield correction was applied by means of images that were observed on the ground of the integrating sphere, which were acquired when the cameras were characterized in the laboratory. In-flight flat-field images are regularly acquired by illuminating the closed telescope front doors with internal lamps. However, internal lamps do not guarantee a completely flat source, and this method is only useful to monitor the flat-field stability, but not to create new reference images. Since no variation was detected on in-flight images, laboratory images are still used for the flat-field correction.

In the WAC images the $16 \mathrm{Cyg} \mathrm{A}+\mathrm{B}$ pair is not separated, which forced us to apply the method to both stars at the same time. In the NAC images the $16 \mathrm{Cyg} \mathrm{A}$ and $\mathrm{B}$ centroids are separated by about ten pixels. This means that we can measure fluxes of 16 Cyg B alone only when the aperture radius is verified to be smaller than 5 pixels, to avoid contamination from $16 \mathrm{Cyg} \mathrm{A}$.

On the CCD, different filters show different typical point spread function (PSF) profiles (see for example Figs. 2 and 3), so that the best aperture radius to use for the aperture photometry depends on the filter, which in many cases is larger than 5 pixels. For consistency between different cameras and among different filters, we therefore decided to derive the photometry from both stars in the NAC in the same way as we have to do with WAC images. See Appendix B for more details on the double star 16 Cyg A+B.

We finally had to define a general criterion to find the best value for the aperture radius. By investigating the stellar integral profile obtained at different aperture radius, we typically expect to find a plateau for aperture radii larger than a critical value after the background level is removed. For OSIRIS images, a typical aperture growth curve of Vega is reported in Fig. 4, where 


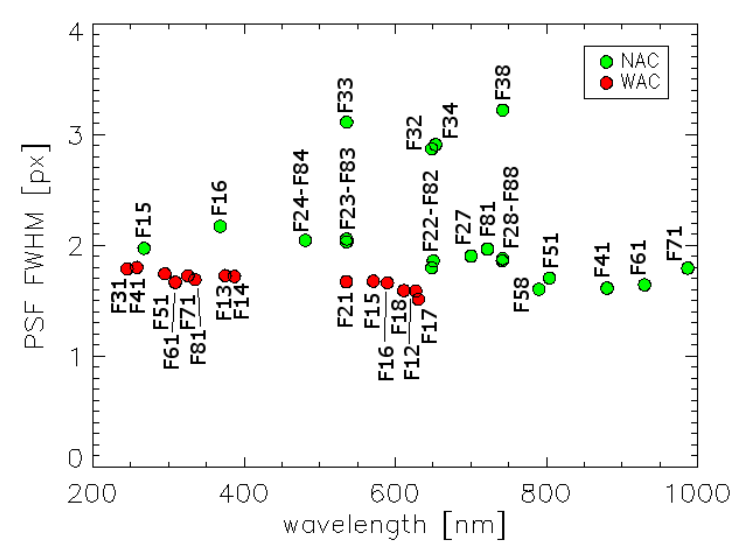

Fig. 2. PSF FWHM as number of pixels (geometric average of $x$ and $y$ direction values) in OSIRIS filters deduced from Vega observations. The largest size of four NAC filters is acquired by combining a near focus plate that can optimize the performances of the NAC camera for a target that is at a distance smaller than $4 \mathrm{~km}$.

the sky level is estimated in a region here defined by sky apertures between 50 and 60 px. For both NAC and WAC, the sky background is normally found to be within $\pm 3 \mathrm{DN}$, with a standard deviation of about $8.5 \mathrm{DN}$ on NAC and 6.7 DN on the WAC, probably due to coherent noise. The plateau indicates that the total flux of the star was taken into account.

In some OSIRIS images this cannot be achieved. We often found an oblique (instead of a horizontal) asymptote, regardless of our definition of the sky annuli. We ascribed this to an onaxis contamination, maybe due to diffuse light by optics microroughness or to ghosts.

The NAC ghosts are well described in Dohlen et al. (2010). First-generation ghosts are caused by the light reflection between pairs of optical surfaces. The most intense on-axis ghost, for example, can be produced by the reflection of the incident light on the surface of the CCD, reflected again by the nearest surface of the ARP. In the [800-1000] nm IR range the NAC CCD reflectivity increases from 10 to $30 \%$. The ghost relative intensity, with respect to the scientific image, is thus three times higher at $1000 \mathrm{~nm}$ than at $800 \mathrm{~nm}$. The two filters most affected by the oblique asymptote are the reddest ones, $F 61$ and $F 71$, which are centered at about 930 and $990 \mathrm{~nm}$.

However, the CCD reflectivity and the ARP reflectivity in the whole [250-400] $\mathrm{nm}$ range are higher than at $1000 \mathrm{~nm}$, which means that the ghost problem should affect the UV range much more than the IR, while we find that the contamination in UV NAC filter, where present, is not so evident. Therefore, further investigation is needed on this topic.

In the meanwhile, we had to find a new definition for the aperture radius for the NAC-F61 and NAC-F71 filters: we selected the smallest aperture radius $r$ such that the flux in the annulus $[r-1, r]$ is below half the sky standard deviation. After that we considered the remnant contribution as background even if with a non-zero average.

\subsubsection{CCD linearity and stability}

We investigated the stability of the results of the aperture photometry method during different observational phases of the mission (from the Mars swing-by in 2007 to the Lutetia fly-by in 2010).
In Table 3 we report the mean value of the count rate computed from Vega images with different filter combinations, the maximum deviation, and the typical exposure times.

For the two NAC (F16 FFP-UV_Near-UV, and F82 Neutral_Orange) and two WAC filters (F12 Red, and F18 Vis610) the signal linearity with increasing exposure time was also investigated. The shortest exposure time is imposed by the shutter response tolerance, the longest time is defined by the CCD saturation level.

The OSIRIS CCDs are equipped with an antiblooming system, designed to "extract" overabundant electrons from the pixel potential well. This system is needed for the cometary target to allow overexposed images of the nucleus without saturation artifacts. This is necessary to acquire high signal-to-noise $(\mathrm{S} / \mathrm{N})$ data of the dust and gas in the inner coma against the brilliant nucleus. The saturation level was required to be about $120000 \mathrm{e}^{-} / \mathrm{px} \approx 40000 \mathrm{DN} / \mathrm{px}$ (see Keller et al. 2007; Thomas et al. 1998) so that the pixel linearity is guaranteed only below this limit. Above the saturation, the CCD cannot collect any other electron, and we will find a constant signal regardless of the exposure time.

For point sources, which are dominated by the optics PSF, the peak value of the source must not exceed the saturation value, while the total flux of the source is split into more pixels and the total number of electrons collected can obviously exceed the limit. When the peak signal reaches the saturation levels, the antiblooming system prevents overabundant charges of only the overexposed pixels to spill over into the neighborhood above a given exposure time. Nearby unsaturated pixels, however, can continue to collect electrons, so that we would still experience an increase of the total point source signal with exposure time, even with a lower rate.

To study the linearity of the CCD response, we defined as outliers and consequently excluded from the data set the count rates that deviate from the mean value by more than three times the standard deviation. The iteration of this method defined a time interval where the linearity is satisfied. Figure 5 shows the distribution of Vega observations (reported as both counts and count rate) vs. exposure time in this interval. In Table 3 the time interval is specified for these four filters.

Table 3 shows that almost all the filters are stable within $\pm 1.5 \%$. In only one case, WAC- $F 51$ at $295 \mathrm{~nm}$, there is a deviation of $+2.79 \%$, while in only two other filters, NAC-F41 at $880 \mathrm{~nm}$ and WAC-F61 at $308.5 \mathrm{~nm}$, deviations exeed $1.5 \%$. Moreover, we also verified that the data considered do not show any evident aging effect since there is no systematic trend with date: the data dispersion of about $\pm 1.5 \%$ is found both in case of stellar observations acquired during the same day and during the whole OSIRIS activity period. This dispersion is also consistent with the photometry precision requirement of about $2 \%$ set by the original mission science goals (Keller et al. 2007).

From this investigation we found that the peak level of counts on a single pixel in raw images is above $57000 \mathrm{DN}$ for both NAC filters investigated, while it is about 47000 and $50000 \mathrm{DN}$ for the two WAC filters investigated, well above the $40000 \mathrm{DN}$ limit foreseen. We verified for WAC that the centroids of the PSF were located in different positions on the CCD, with different efficiencies: if the saturation limit was found to be at $47000 \mathrm{DN}$, the star always fell on some few cold pixels. This difference was then corrected by flat fielding. The difference between NAC and WAC values suggest that the two CCDs behave differently. For NAC-F82 we did not exclude any observation in the data-set with the longest exposure times, so that we did not confirme the saturation limit in this case. For new tests that are scheduled to 
a)

NAC-F16

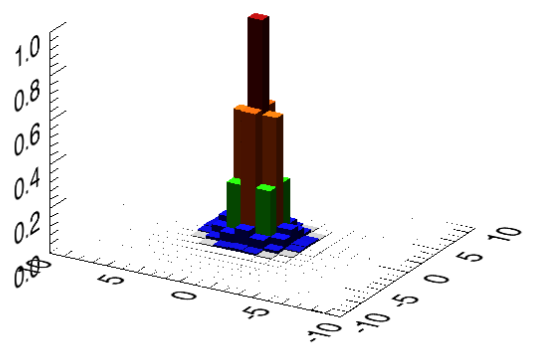

c)
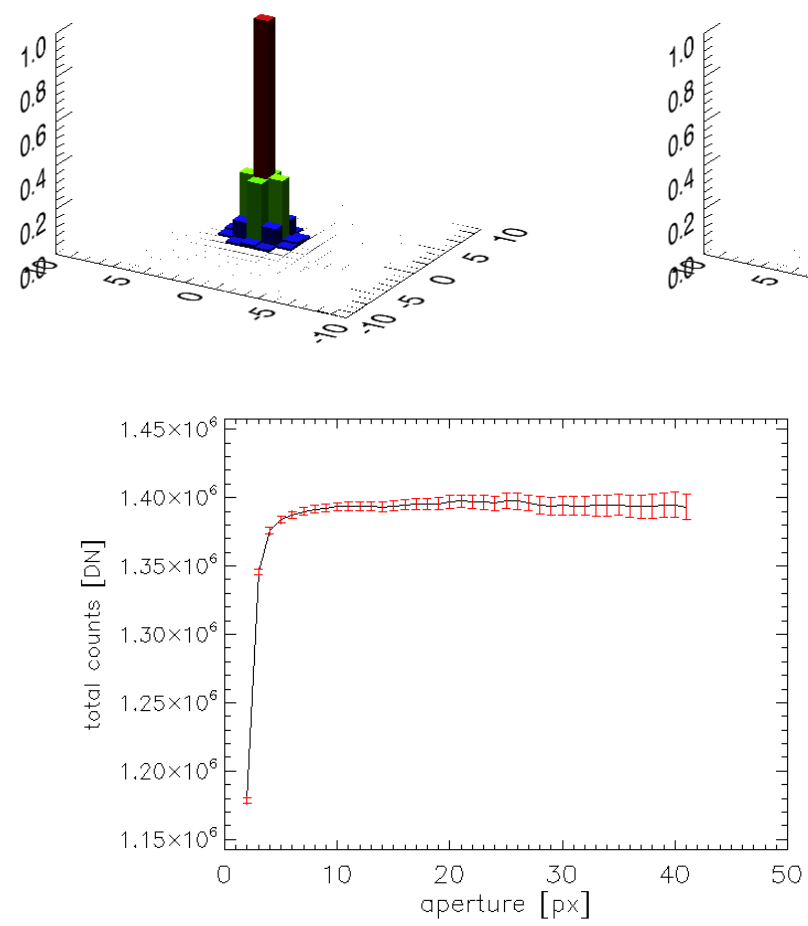

Fig. 4. Typical curve of the aperture flux growth. This curve was specifically obtained from Vega observations with the NAC F16 filter.

study the CCD linearity, a wider range in $t_{\exp }$ for NAC-F82 must be considered.

In Fig. 3 we show the PSF for two NAC and two WAC filters in the case where the centroid of the PSF lies exactly on the center of a pixel. For the four selected filters the ratios between the peak value and the total integrated signal are about $17.5 \%$ for NAC-F16, $24.5 \%$ for NAC-F82, 34.5\% for WAC- $F 12$, and $34.3 \%$ for WAC-F 18 of the total signal.

\subsection{Count rate: observed vs. expected}

In Fig. 6 we show the ratio between observed and expected counts in each filter for Vega and $16 \mathrm{Cyg} \mathrm{A}+\mathrm{B}$. The count rate obtained for Vega and 16 Cyg A+B observations acquired during all mission phases was compared with the expected rate that we computed from the Vega reference spectrum (Bohlin \& Gilliland 2004, CALSPEC version 004) and solar reference spectra (Burlov-Vasiljev et al. 1995, 1998), respectively, by means of Eq. (1). For this last source we computed a conversion factor of between $16 \mathrm{Cyg} \mathrm{A}+\mathrm{B}$ and solar fluxes, based on the

\section{NAC-F82}

Fig. 3. Shape of the Moffat fit of the PSF of OSIRIS camera filters in the following combinations: a) NAC-F16 (FFP-UV_Near-UV); b) NAC-F82 (Neutral_Orange); c) WAC-F12 (Red); and d) WAC-F18 (Vis610).

different visual absolute magnitudes, to allow a direct comparison with the solar spectrum. See Appendix B for further details.

The error bars in this plot were computed by propagating the photon noise deduced on the original images with errors defined on each instrument element. Horizontal bars refer to the FWHM of each passband.

The total relative (percentage) instrumental error is dominated by the CCD QE uncertainty.

Several considerations arise from the analysis of the plot:

- In the UV range, that is, below $400 \mathrm{~nm}$, fewer counts are observed than expected.

- In the visible range, the WAC filters counts agree with the predicted counts. The NAC filter counts, however, seem to exceed the expected counts (in some cases they are even higher than the vertical error bars).

- In the near-IR range, above $800 \mathrm{~nm}$, fewer counts are observed counts than predicted.

- The NAC twin filter values agree within error bars. However, we found some systematic differences among NAC FFP-Vis, NAC NFP-Vis, and NAC Neutral (second, third, and eighth position on filter wheel 1 , respectively).

- The agreement between observed and expected counts is verified from both Vega and $16 \mathrm{Cyg} \mathrm{A}+\mathrm{B}$ counts in the range $470-890 \mathrm{~nm}$.

- The agreement between Vega and $16 \mathrm{Cyg} \mathrm{A}+\mathrm{B}$ data is good above $470 \mathrm{~nm}$.

- The scatter among filters and between different stars is larger in the UV range. $16 \mathrm{Cyg} \mathrm{A}+\mathrm{B}$ data are systematically lower than Vega data.

- Around WAC F71 (centered on $325 \mathrm{~nm}$ ) the plot behaves strangely in both Vega and $16 \mathrm{Cyg} \mathrm{A}+\mathrm{B}$ data. This scatter is not due to an anomalous observation because the photometric values were computed for this filter, like for others, as the average of many observation in different phases, giving a positive and a negative maximum percentage error of $+1.11 \%$ and $-1.36 \%$, respectively. 
Table 3. OSIRIS NAC and WAC filter characteristics and Vega observations.

\begin{tabular}{|c|c|c|c|c|c|c|c|c|}
\hline Filter & $\begin{array}{c}\text { Central } \lambda \\
\mathrm{nm}\end{array}$ & $\begin{array}{c}F W H M \\
\mathrm{~nm}\end{array}$ & $\begin{array}{c}T_{\exp } \\
\mathrm{S}\end{array}$ & $\begin{array}{c}\text { Mean count rate } \\
\text { DN/s }\end{array}$ & $\begin{array}{c}+ \text { err } \\
\%\end{array}$ & $\begin{array}{l}\text {-err } \\
\%\end{array}$ & Observations & $\begin{array}{c}\text { Aperture } \\
\text { px }\end{array}$ \\
\hline NAC-F15 & 267.5 & 53 & 0.400 & $2.571 \mathrm{e}+005$ & 1.07 & 0.95 & 6 & $6-8$ \\
\hline NAC-F16 & 368.5 & 33 & $0.020-0.256$ & $1.415 e+006$ & 0.59 & 0.75 & 30 & $8-10^{*}$ \\
\hline NAC- $F 22$ & 648.5 & 83 & 0.020 & $7.172 \mathrm{e}+006$ & 1.19 & 0.93 & 5 & $9-11$ \\
\hline NAC-F23 & 535.5 & 61 & 0.020 & $7.265 e+006$ & 0.80 & 0.69 & 5 & $10-12$ \\
\hline NAC-F 24 & 481.0 & 72 & 0.020 & $9.369 \mathrm{e}+006$ & 0.15 & 0.30 & 5 & $10-11$ \\
\hline NAC- $F 27$ & 700.5 & 21 & 0.080 & $1.366 \mathrm{e}+006$ & 1.29 & 0.91 & 5 & $9-10$ \\
\hline NAC- $F 28$ & 742.0 & 62 & 0.025 & $3.447 \mathrm{e}+006$ & 0.27 & 0.22 & 5 & $9-11$ \\
\hline NAC-F32 & 648.5 & 83 & 0.040 & $7.496 \mathrm{e}+006$ & 0.41 & 0.83 & 5 & $13-14$ \\
\hline NAC-F33 & 535.5 & 61 & 0.040 & $7.581 \mathrm{e}+006$ & 1.38 & 1.25 & 5 & $13-15$ \\
\hline NAC-F38 & 742.0 & 62 & 0.060 & $3.565 e+006$ & 1.10 & 0.61 & 4 & $13-14$ \\
\hline NAC-F41 & 880.0 & 62 & 0.100 & $9.906 e+005$ & 1.76 & 1.03 & 5 & $10-11$ \\
\hline NAC-F51 & 804.5 & 39 & 0.100 & $9.895 e+005$ & 1.16 & 0.79 & 5 & $9-10$ \\
\hline NAC- $F 58$ & 790.5 & 23 & 1.000 & $7.631 \mathrm{e}+004$ & 0.50 & 0.34 & 6 & $9-10$ \\
\hline NAC-F61 & 929.5 & 39 & 0.350 & $3.223 e+005$ & 0.71 & 1.15 & 5 & $13-15$ \\
\hline NAC-F71 & 987.0 & 38 & 0.800 & $1.092 \mathrm{e}+005$ & 1.43 & 1.50 & 5 & $16-17$ \\
\hline NAC-F82 & 650.5 & 81 & $0.070-1.297$ & $1.941 \mathrm{e}+005$ & 0.58 & 0.76 & 32 & $9-11^{*}$ \\
\hline NAC- $F 83$ & 535.5 & 59 & 0.500 & $2.033 e+005$ & 1.32 & 0.80 & 5 & $8-9$ \\
\hline NAC- $F 88$ & 742.0 & 60 & 0.500 & $1.752 \mathrm{e}+005$ & 1.37 & 1.43 & 5 & $9-10$ \\
\hline WAC- $F 12$ & 627.0 & 154 & $0.010-0.150$ & $1.169 \mathrm{e}+006$ & 1.39 & 1.35 & 66 & $8-11 *$ \\
\hline WAC-F13 & 375.0 & 8 & 6.000 & $1.875 \mathrm{e}+004$ & 1.23 & 0.85 & 8 & $11-12$ \\
\hline WAC-F14 & 387.0 & 4 & 6.000 & $1.588 \mathrm{e}+004$ & 1.21 & 0.93 & 9 & $10-12$ \\
\hline WAC-F15 & 571.0 & 10 & 1.500 & $8.142 \mathrm{e}+004$ & 0.73 & 0.53 & 9 & $9-11$ \\
\hline WAC-F16 & 589.5 & 3 & 3.500 & $3.030 \mathrm{e}+004$ & 0.77 & 0.55 & 8 & $9-10$ \\
\hline WAC-F17 & 630.5 & 3 & 3.500 & $2.029 \mathrm{e}+004$ & 0.85 & 0.97 & 9 & $7-9$ \\
\hline WAC-F18 & 611.5 & 9 & $0.233-1.900$ & $8.288 \mathrm{e}+004$ & 0.52 & 1.14 & 29 & $8-10 *$ \\
\hline WAC- $F 21$ & 535.5 & 61 & 0.200 & $5.910 \mathrm{e}+005$ & 0.68 & 0.28 & 9 & $9-11$ \\
\hline WAC-F31 & 245.5 & 13 & 15.000 & $5.308 \mathrm{e}+003$ & 0.85 & 1.27 & 9 & $12-13$ \\
\hline WAC-F41 & 258.0 & 4 & 50.000 & $1.749 \mathrm{e}+003$ & 1.05 & 0.84 & 8 & $12-14$ \\
\hline WAC- $F 51$ & 295.0 & 10 & 25.000 & $3.060 \mathrm{e}+003$ & 2.79 & 1.79 & 6 & $12-13$ \\
\hline WAC-F61 & 308.5 & 3 & 60.000 & $1.273 \mathrm{e}+003$ & 1.77 & 1.85 & 8 & $11-13$ \\
\hline WAC-F71 & 325.5 & 9 & 20.000 & $4.376 e+003$ & 1.11 & 1.36 & 9 & $12-13$ \\
\hline WAC- $F 81$ & 335.0 & 4 & 80.000 & $1.422 \mathrm{e}+003$ & 0.86 & 0.69 & 8 & $11-12$ \\
\hline
\end{tabular}

Notes. This table refers to the observations of Vega during different mission phases, from the Mars swing-by in 2007 to the Lutetia fly-by in 2010. For each filter we report the central wavelength of the whole instrumental and filter passband in nm; the FWHM of each instrumental and filter passband in $\mathrm{nm}$; the exposure time needed for the Vega observation, and for the four investigated filters, the linearity interval in seconds; the average count rate in $\mathrm{DN} \mathrm{s}^{-1}$ for Vega observations; the positive and negative maximum deviation (expressed in \%) with respect to the mean value; the total number of observations; the typical values of the apertures among all observations; ${ }^{(*)}$ for each of the four additional filters, this refers only to observations performed with a single particular exposure time within the interval specified in the fourth column.

16 Cyg A+B data can be affected by an incorrect computation of the conversion from solar fluxes (computed from $\left.10^{-0.4\left(V_{\mathrm{SA}}-V_{\odot}\right)}\right)$. However, the match of $16 \mathrm{Cyg} \mathrm{A}+\mathrm{B}$ data with Vega data above $470 \mathrm{~nm}$ suggests that this assumption is good at least in the visible and near-infrared range of the spectrum. In the UV range, there may be also some differences due to the imperfect match between 16 Cyg A and 16 Cyg B spectra and the solar spectrum, which can be variable in these wavelengths by up to $20 \%$ (Colina et al. 1996). Moreover, below $325 \mathrm{~nm}, 16$ Cyg A+B counts are maybe unreliable as a result of the decreasing signal in this wavelength range that is typical of solar analog stars, which means that we can only trust the Vega counts.

Variability among twin filters that share the same passband (i.e., NAC F82-F22-F32, NAC F83-F23-F33, NAC F88-F28$F 38$, etc.) are probably due to different shapes of the actual transmission curves of clear filters (NAC FFP-Vis, NAC NFP-Vis, and NAC Neutral) with respect to laboratory data of the same filters obtained on the ground before the launch.

The lower count rate in the near IR can in principle be due to an incorrect computation of the counts within the selected aperture. For many NAC filters the aperture radii selected for the computation of Vega flux through the aperture photometry method were found to be 7-9 pixels. We recall here that for these filters we always found a plateau. However, throughout all the past mission phases, the signal was always found to increase for the NAC IR filters F61 and F71; it lay on an oblique asymptote. Taking into account a possible on-axis contamination, we decided to stop at an aperture of about 10-12 pixels for $F 61$ and at $15-17$ pixels for $F 71$, depending on the background noise level on the different images. For the sake of completeness, we investigated wider apertures for the NAC-F61 and F71 filters. We found that even 40-pixel apertures are not enough to obtain the expected counts, hence wider apertures cannot improve the calibration in either case.

\section{Absolute flux calibration}

We call the factor needed to convert observed count rates per pixel into physical units the abscal factor (i.e., spectral radiance, expressed in $\mathrm{W} \mathrm{m}^{-2} \mathrm{~nm}^{-1} \mathrm{sr}^{-1}$ ) to obtain the absolute instrument calibration.

We present photometric calibration factors for each passband. We used images of stellar targets such as Vega and the pair $16 \mathrm{Cyg} \mathrm{A}+\mathrm{B}$. According to each filter combination $(x, y)$, 
S. Magrin et al.: Pre-hibernation performances of the OSIRIS cameras onboard the Rosetta spacecraft
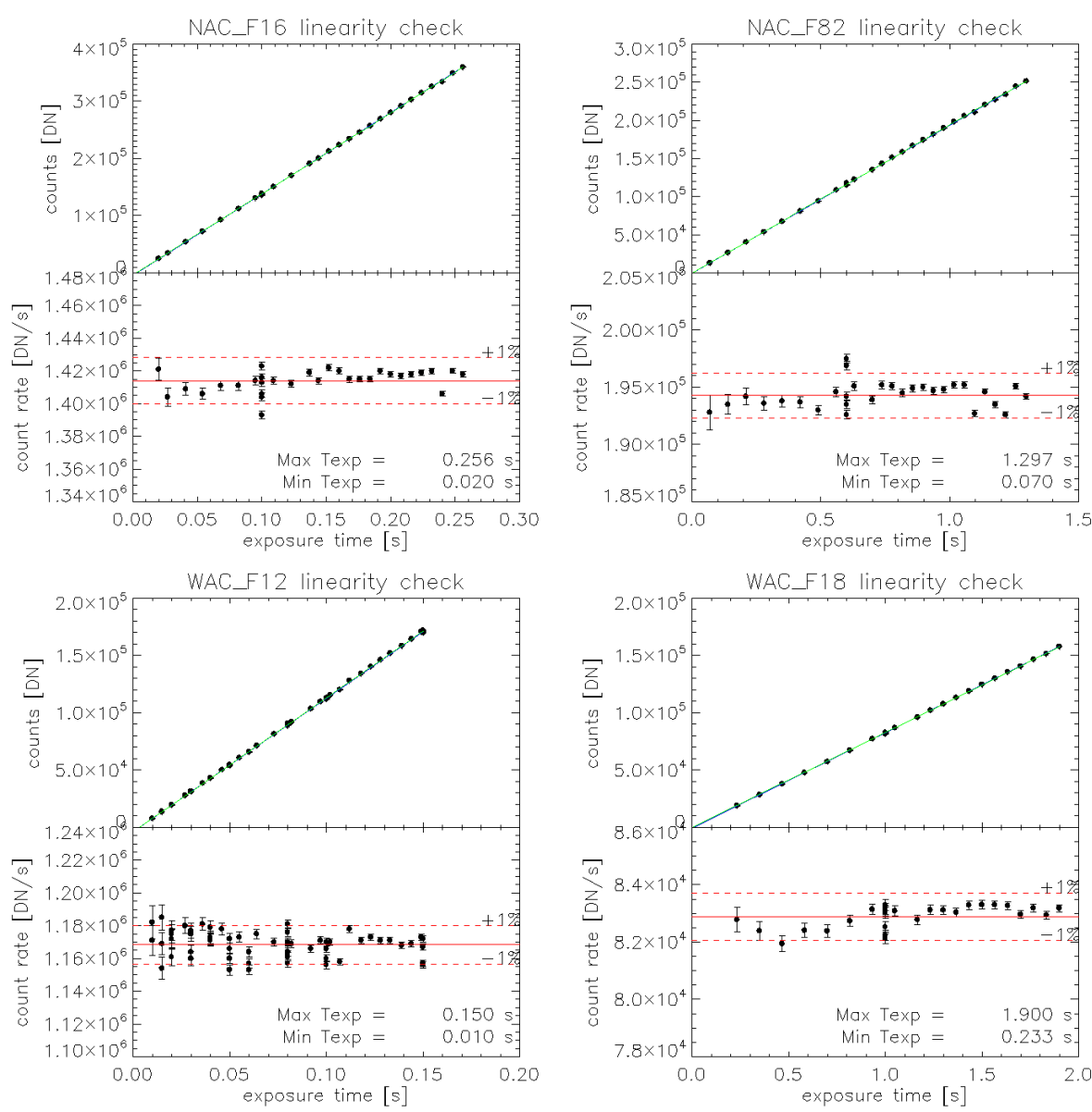

Fig. 5. Linearity measurements obtained by the Vega observations in terms of DN or DN/s vs exposure time. For each filter, the upper panels show counts vs. exposure time in the particular filter, the lower panels show the count rate vs. the exposure time. The investigation was made with the following filters: a) NAC-F16 (FFP-UV_Near-UV); b) NAC-F82 (Neutral_Orange); c) WAC-F12 (Red); and d) WAC-F18 (Vis610).
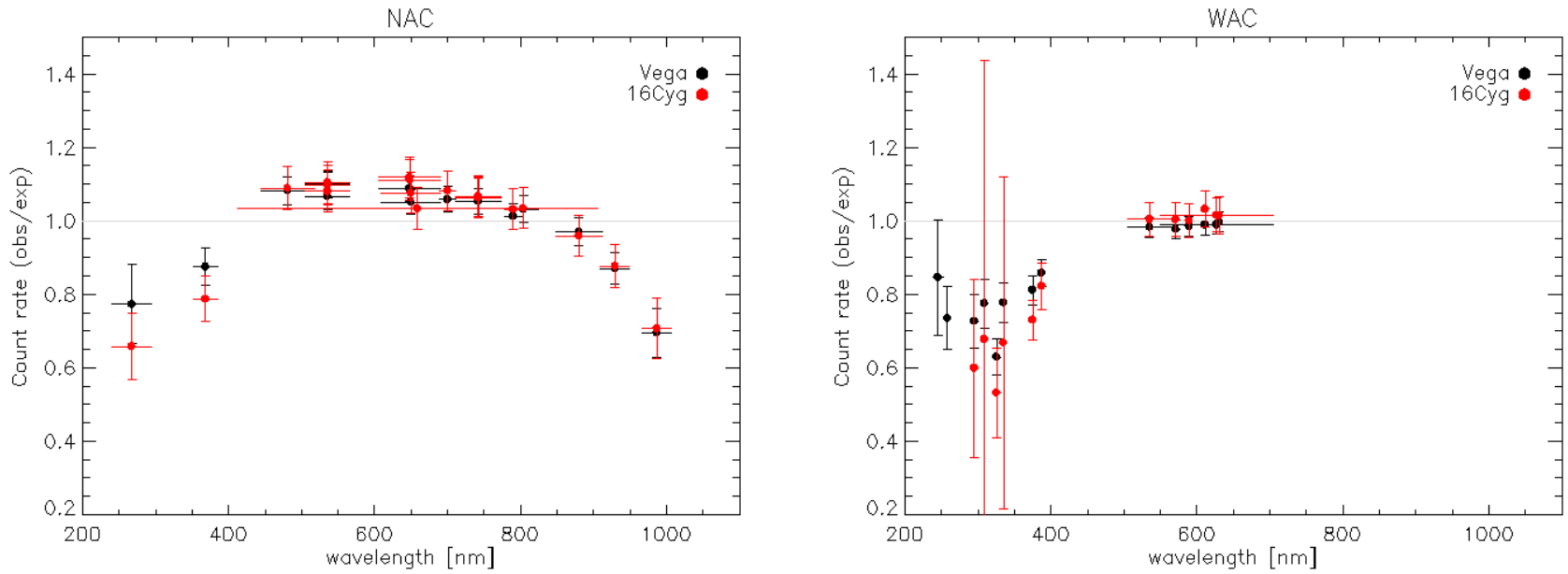

Fig. 6. Ratio between observed and expected counts from the CCD with a particular filter setting. Vertical error bars refer to photon noise and the propagation of instrumental uncertainties. Horizontal bars refer to the FWHM of each passband. Red data refer to counts computed from 16 Cyg A+B images and solar spectrum (Burlov-Vasiljev et al. 1995, 1998) scaled to 16 Cyg A+B magnitude; black data refer to Vega images and Vega spectrum (Bohlin \& Gilliland 2004).

we can measure the total count rate due to the star (or pair), $K_{x, y}$, from these images.

The spectral radiance that we expect on each pixel with the same filter combination $(x, y)$ is the weighted average of the incoming spectral radiance of the target star, with the instrumental response as the weight, divided by the pixel size (pxsz) of the CCD expressed in steradians (3.547e-010 sr for NAC and 9.982e-009 sr for WAC):

$$
\phi_{x, y}=\frac{\int_{0}^{+\infty} \varphi(\lambda) M^{n}(\lambda) F_{x, y}(\lambda) T_{\mathrm{ARP}}(\lambda) Q(\lambda) \frac{\lambda}{h c} \frac{\mathrm{AP}}{\mathrm{IG}} \mathrm{d} \lambda}{\operatorname{pxsz} \int_{0}^{+\infty} M^{n}(\lambda) F_{x, y}(\lambda) T_{\mathrm{ARP}}(\lambda) Q(\lambda) \frac{\lambda}{h c} \frac{\mathrm{AP}}{\mathrm{IG}} \mathrm{d} \lambda}
$$


Table 4. Absolute flux calibration of the OSIRIS NAC and WAC filters.

\begin{tabular}{|c|c|c|c|c|c|c|c|c|}
\hline Filter & $\begin{array}{c}\text { Central } \lambda \\
\mathrm{nm}\end{array}$ & $\begin{array}{c}F W H M \\
\mathrm{~nm}\end{array}$ & $\begin{array}{c}\text { Abscal factor } \\
\mathrm{DN} \mathrm{s}^{-1} \cdot\left(\mathrm{Wm}^{-2} \mathrm{~nm}^{-1} \mathrm{sr}^{-1}\right)^{-1}\end{array}$ & $\begin{array}{c}+ \text { err } \\
\%\end{array}$ & $\begin{array}{c}- \text { err } \\
\%\end{array}$ & $\begin{array}{l}\text { Abscal reflectance factor } \\
\mathrm{DN} \mathrm{s}^{-1}\end{array}$ & $\begin{array}{c}+ \text { err } \\
\%\end{array}$ & $\begin{array}{c}- \text { err } \\
\%\end{array}$ \\
\hline NAC F15 & 267.5 & 53.0 & $2.428 \mathrm{e}+006$ & 16.7 & 16.7 & $1.434 \mathrm{e}+005$ & 15.3 & 15.3 \\
\hline NAC F16 & 368.5 & 33.0 & $1.308 \mathrm{e}+007$ & 5.6 & 5.7 & $4.566 e+006$ & 5.6 & 5.7 \\
\hline NAC F21 & 659.0 & 492.0 & $6.190 \mathrm{e}+008$ & 3.3 & 3.2 & $2.830 \mathrm{e}+008$ & 1.7 & 1.7 \\
\hline NAC $F 22$ & 648.5 & 83.0 & $1.182 \mathrm{e}+008$ & 2.5 & 2.4 & $5.892 \mathrm{e}+007$ & 2.5 & 2.4 \\
\hline NAC $F 23$ & 535.5 & 61.0 & $6.677 \mathrm{e}+007$ & 2.4 & 2.5 & $4.01 \mathrm{e}+007$ & 2.4 & 2.5 \\
\hline NAC $F 24$ & 481.0 & 72.0 & $6.421 \mathrm{e}+007$ & 2.7 & 2.5 & $4.086 e+007$ & 2.7 & 2.5 \\
\hline NAC $F 26$ & 368.5 & 33.0 & $1.232 \mathrm{e}+007$ & 5.6 & 5.7 & $4.307 e+006$ & 5.6 & 5.7 \\
\hline NAC $F 27$ & 700.5 & 21.0 & $2.824 \mathrm{e}+007$ & 2.4 & 2.4 & $1.270 \mathrm{e}+007$ & 2.4 & 2.4 \\
\hline NAC $F 28$ & 742.0 & 62.0 & $8.544 \mathrm{e}+007$ & 2.3 & 2.3 & $3.495 \mathrm{e}+007$ & 2.3 & 2.3 \\
\hline NAC F31 & 653.5 & 479.0 & $6.297 \mathrm{e}+008$ & 3.2 & 3.2 & $2.907 e+008$ & 3.9 & 3.9 \\
\hline NAC F32 & 648.5 & 83.0 & $1.235 \mathrm{e}+008$ & 2.5 & 2.4 & $6.156 e+007$ & 2.5 & 2.4 \\
\hline NAC $F 33$ & 535.5 & 61.0 & $6.959 e+007$ & 2.5 & 2.6 & $4.169 \mathrm{e}+007$ & 2.5 & 2.6 \\
\hline NAC $F 34$ & 481.0 & 72.0 & $6.741 \mathrm{e}+007$ & 2.6 & 2.5 & $4.290 \mathrm{e}+007$ & 2.6 & 2.5 \\
\hline NAC $F 35$ & 289.5 & 13.0 & $3.157 \mathrm{e}+005$ & 14.9 & 14.9 & $3.980 \mathrm{e}+004$ & 14.5 & 14.5 \\
\hline NAC $F 36$ & 371.5 & 27.0 & $1.071 \mathrm{e}+007$ & 5.6 & 5.7 & $3.765 e+006$ & 5.5 & 5.6 \\
\hline NAC F37 & 700.5 & 21.0 & $2.942 \mathrm{e}+007$ & 2.4 & 2.4 & $1.323 e+007$ & 2.4 & 2.4 \\
\hline NAC $F 38$ & 742.0 & 62.0 & $8.842 \mathrm{e}+007$ & 2.3 & 2.3 & $3.618 \mathrm{e}+007$ & 2.3 & 2.3 \\
\hline NAC F41 & 880.0 & 62.0 & $4.024 \mathrm{e}+007$ & 3.4 & 3.2 & $1.209 e+007$ & 3.4 & 3.2 \\
\hline NAC F51 & 804.5 & 39.0 & $3.159 e+007$ & 2.7 & 2.5 & $1.141 \mathrm{e}+007$ & 2.7 & 2.5 \\
\hline NAC $F 58$ & 790.5 & 23.0 & $2.326 e+006$ & 2.9 & 2.5 & $8.626 e+005$ & 2.9 & 2.5 \\
\hline NAC F61 & 929.5 & 39.0 & $1.444 \mathrm{e}+007$ & 4.3 & 4.3 & $3.894 \mathrm{e}+006$ & 4.3 & 4.3 \\
\hline NAC $F 71$ & 987.0 & 38.0 & $5.763 e+006$ & 10.8 & 10.7 & $1.384 \mathrm{e}+006$ & 10.7 & 10.6 \\
\hline NAC $F 81$ & 722.0 & 206.0 & $1.937 \mathrm{e}+007$ & 3.1 & 3.1 & $8.938 \mathrm{e}+006$ & 3.8 & 3.8 \\
\hline NAC $F 82$ & 650.5 & 81.0 & $3.246 \mathrm{e}+006$ & 2.7 & 2.4 & $1.607 e+006$ & 2.7 & 2.4 \\
\hline NAC $F 83$ & 535.5 & 59.0 & $1.869 \mathrm{e}+006$ & 2.5 & 2.4 & $1.120 \mathrm{e}+006$ & 2.5 & 2.4 \\
\hline NAC F84 & 481.0 & 72.0 & $1.991 \mathrm{e}+006$ & 2.7 & 2.5 & $1.270 \mathrm{e}+006$ & 2.7 & 2.5 \\
\hline NAC $F 86$ & 378.0 & 18.0 & $4.980 \mathrm{e}+004$ & 5.1 & 5.2 & $1.819 \mathrm{e}+004$ & 5.0 & 5.1 \\
\hline NAC F87 & 700.5 & 21.0 & $1.313 \mathrm{e}+006$ & 2.4 & 2.4 & $5.891 \mathrm{e}+005$ & 2.4 & 2.4 \\
\hline NAC $F 88$ & 742.0 & 60.0 & $4.286 e+006$ & 2.7 & 2.8 & $1.756 e+006$ & 2.7 & 2.8 \\
\hline WAC F12 & 627.0 & 154.0 & $4.810 \mathrm{e}+008$ & 2.5 & 2.6 & $2.52 \mathrm{e}+008$ & 2.5 & 2.6 \\
\hline WAC F13 & 375.0 & 8.0 & $4.684 \mathrm{e}+006$ & 4.8 & 4.8 & $1.774 \mathrm{e}+006$ & 4.8 & 4.8 \\
\hline WAC F14 & 387.0 & 4.0 & $2.538 \mathrm{e}+006$ & 3.8 & 3.9 & $8.826 \mathrm{e}+005$ & 3.8 & 3.8 \\
\hline WAC F 15 & 571.0 & 10.0 & $2.557 \mathrm{e}+007$ & 2.3 & 2.1 & $1.494 \mathrm{e}+007$ & 2.3 & 2.1 \\
\hline WAC F16 & 589.5 & 3.0 & $1.045 \mathrm{e}+007$ & 2.1 & 2.1 & $5.823 e+006$ & 2.1 & 2.1 \\
\hline WAC F17 & 630.5 & 3.0 & $8.594 \mathrm{e}+006$ & 2.1 & 2.3 & $4.435 \mathrm{e}+006$ & 2.1 & 2.3 \\
\hline WAC $F 18$ & 611.5 & 9.0 & $3.196 e+007$ & 2.1 & 2.2 & $1.724 \mathrm{e}+007$ & 2.1 & 2.2 \\
\hline WAC $F 21$ & 535.5 & 61.0 & $1.523 e+008$ & 2.3 & 2.1 & $9.131 \mathrm{e}+007$ & 2.3 & 2.1 \\
\hline WAC F31 & 245.5 & 13.0 & $1.370 \mathrm{e}+006$ & 23.6 & 23.5 & $3.449 \mathrm{e}+004$ & 20.9 & 20.7 \\
\hline WAC F41 & 258.0 & 4.0 & $4.686 \mathrm{e}+005$ & 13.9 & 13.8 & $1.952 \mathrm{e}+004$ & 12.9 & 12.8 \\
\hline WAC F51 & 295.0 & 10.0 & $8.483 e+005$ & 11.8 & 11.7 & $1.443 \mathrm{e}+005$ & 11.7 & 11.6 \\
\hline WAC F61 & 308.5 & 3.0 & $3.592 \mathrm{e}+005$ & 9.8 & 9.7 & $7.412 \mathrm{e}+004$ & 9.6 & 9.5 \\
\hline WAC $F 71$ & 325.5 & 9.0 & $1.312 \mathrm{e}+006$ & 8.6 & 8.6 & $3.690 \mathrm{e}+005$ & 8.6 & 8.5 \\
\hline WAC $F 81$ & 335.0 & 4.0 & $4.447 \mathrm{e}+005$ & 7.7 & 7.6 & $1.357 \mathrm{e}+005$ & 7.7 & 7.6 \\
\hline
\end{tabular}

Notes. In this table we report the absolute flux and reflectance calibration of all OSIRIS NAC and WAC filter combinations, obtained using the whole data set of Vega and 16 Cyg A+B observations during the different mission phases from the Mars swing-by in 2007 to the Lutetia fly-by in 2010. For each filter we report the central wavelength of the whole instrumental and filter passband in nm; the FWHM of each instrumental and filter passband in $\mathrm{nm}$; the abscal factor needed to convert the image count rate into spectral radiance; its positive and negative percentage error; the abscal reflectance factor needed to convert the image count rate into reflectance; its positive and negative percentage error.

Consequently, the abscal factor, $A_{x, y}$, which links the total count rate $\left(K_{x, y}\right)$ on the $\mathrm{CCD}$ for a particular filter combination to the spectral radiance within the passbands, $\phi_{x, y}$, expressed in $\mathrm{W} \mathrm{m} \mathrm{mm}^{-1} \mathrm{sr}^{-1}$, is defined by

$A_{x, y}=K_{x, y} / \phi_{x, y}$.

In first approximation $A_{x, y}$ is filter dependent (and hence in principle time (age) dependent) and star independent.

Because AP, $h c$, and IG are constant with wavelength and can be simplified, the abscal factor is

$A_{x, y}=\frac{K_{x, y} \operatorname{pxsz} \int_{0}^{+\infty} M^{n}(\lambda) F_{x, y}(\lambda) T_{\mathrm{ARP}}(\lambda) Q(\lambda) \lambda \mathrm{d} \lambda}{\int_{0}^{+\infty} \varphi(\lambda) M^{n}(\lambda) F_{x, y}(\lambda) T_{\mathrm{ARP}}(\lambda) Q(\lambda) \lambda \mathrm{d} \lambda}$.
The abscal factor is expressed in $\mathrm{DN} \mathrm{s}^{-1} \cdot\left(\mathrm{Wm}^{-2} \mathrm{~nm}^{-1} \mathrm{sr}^{-1}\right)^{-1}$.

In Table 4 we report the abscal factor values for all different filters computed from this equation.

$Q(\lambda)$ is roughly constant on the visible passbands and can be simplified from both integrals in Eq. (3): this means in particular that the abscal factors are little affected by the imperfect knowledge of the CCD quantum efficiency in the visible range (approximately [400-1000] nm).

\subsection{Twin combinations}

As said in the Introduction, we can calibrate scientific images acquired with any of the filters of a twin group by using 
Table 5. List of NAC filters without stellar calibration.

\begin{tabular}{ccc}
\hline \hline Camera & $\begin{array}{c}\text { Filter } \\
\text { to calibrate }\end{array}$ & $\begin{array}{c}\text { Baseline } \\
\text { filter }\end{array}$ \\
\hline NAC & 26 & 16 \\
NAC & 31 & 21 \\
NAC & 34 & 24 \\
NAC & 35 & 15 \\
NAC & 36 & 16 \\
NAC & 37 & 27 \\
NAC & 81 & 21 \\
NAC & 84 & 24 \\
NAC & 86 & 16 \\
NAC & 87 & 27 \\
\hline
\end{tabular}

Notes. To calibrate these filters, we used the count rate from the baseline filters listed in the right column.

the baseline calibration of a representative filter of the group. For example, we can calibrate both NAC-F87 and NAC-F37, or NAC-F84 and NAC-F34, simply by using the baseline calibration obtained with filter combinations NAC $F 27$ or NAC $F 24$, respectively. In Table 5 we show all the filters for which this is possible and the selected baseline filter.

In the following we describe the equations used to properly infer the absolute calibration in these cases. As an example, we use the calibration of NAC-F84 images through the NAC-F24 observation of the calibration star. The filter in the second position (2) on the NAC first filter wheel (FFP-Vis: far focus plate - visible) has a mean efficiency $\approx 95 \%$, while filter 8 (neutral) has $\approx 5 \%$. Accordingly, the two considered filters refer to Neutral_Blue or FFP-Vis_Blue (coded as NAC-F84 or and NAC-F24), respectively. To compute the abscal factor of the filter combination $A_{84}$, we cannot use the usual expression

$A_{84}=\frac{K_{84} \operatorname{pxsz} \int_{0}^{+\infty} M^{n}(\lambda) F_{84}(\lambda) T_{\mathrm{ARP}}(\lambda) Q(\lambda) \lambda \mathrm{d} \lambda}{\int_{0}^{+\infty} \varphi(\lambda) M^{n}(\lambda) F_{84}(\lambda) T_{\mathrm{ARP}}(\lambda) Q(\lambda) \lambda \mathrm{d} \lambda}$

since we did not observe calibration stars through the $F_{84}$ filter, we do not know the $K_{84}$ count rate, but only $K_{24}$. However, we expect from Eq. (1) that the counts of a star through a filter are proportional to the integral of the stellar spectrum multiplied by the passbands, so that

$\frac{K_{84}}{K_{24}}=\frac{\int_{0}^{+\infty} \varphi(\lambda) M^{n}(\lambda) T_{\mathrm{ARP}}(\lambda) Q(\lambda) F_{84}(\lambda) \lambda \mathrm{d} \lambda}{\int_{0}^{+\infty} \varphi(\lambda) M^{n}(\lambda) T_{\mathrm{ARP}}(\lambda) Q(\lambda) F_{24}(\lambda) \lambda \mathrm{d} \lambda}$.

This means that we can express $A_{84}$ as

$A_{84}=K_{24} \frac{\operatorname{pxsz} \int_{0}^{+\infty} M^{n}(\lambda) F_{84}(\lambda) T_{\mathrm{ARP}}(\lambda) Q(\lambda) \lambda \mathrm{d} \lambda}{\int_{0}^{+\infty} \varphi(\lambda) M^{n}(\lambda) F_{24}(\lambda) T_{\mathrm{ARP}}(\lambda) Q(\lambda) \lambda \mathrm{d} \lambda}$.

This method was applied to complete the absolute flux calibration presented in Table 4 for filters without a direct observation of the calibration stars.

\subsection{Theoretical and real abscal factor}

Since the expected counts from a source should be expressed as in Eq. (1), the theoretic abscal factor should also be expressed as

$A_{x, y \text { theoretic }}=\operatorname{pxsz} \int_{0}^{+\infty} M^{n}(\lambda) F_{x, y}(\lambda) T_{\mathrm{ARP}}(\lambda) Q(\lambda) \frac{\lambda}{h c} \frac{\mathrm{AP}}{\mathrm{IG}} \mathrm{d} \lambda$.
In this form we can see that the abscal factor should be calibrator independent. However, the expected counts in general may be not equal to the observed counts, as shown in Sect. 2.3. This is why the previous formulation is only the theoretical formulation and needs to be corrected for each variation on the instrumental response with time (age).

In the following, we call $C_{x, y}$ the ratio between observed and expected counts in the $(x, y)$ filter. It is defined by

$C_{x, y}=\frac{K_{x, y \text { observed }}}{\int_{0}^{+\infty} \varphi(\lambda) M^{n}(\lambda) F_{x, y}(\lambda) T_{\mathrm{ARP}}(\lambda) Q(\lambda) \frac{\lambda}{h c} \frac{\mathrm{AP}}{\mathrm{IG}} \mathrm{d} \lambda}$.

This equation defines the correction factor that we need to apply to the optics profiles so that Eq. (1) gives the observed count rate. However, with this definition we only assume single correction factors, constant on wavelengths, for each $(x, y)$ filter combination.

In this approximation the abscal factor we should use to convert our images becomes

$A_{x, y \text { real }} \cong C_{x, y} A_{x, y \text { theoretic }}$

We stress that this is not an exact equivalence. We should not only correct for $C_{x, y}$ factors, constant on wavelength, but we should find a correction profile $C(\lambda)$, dependent on the wavelength, deduced by $C_{x, y}$ data, that can correct the shape of every passband profile.

However, where the response diversity is a constant factor over the passband (or maybe even a slow symmetrical variation over the passband), the theoretical source flux weighted mean is the same as the observed one, and the previous equivalence is exact. This is verified for OSIRIS filters in the [470-900] nm range.

In this case, we can write

$$
\begin{aligned}
A_{x, y \text { real }}= & K_{x, y \text { observed pxsz }} \\
& \times \frac{\int_{0}^{+\infty} M^{n}(\lambda) F_{x, y}(\lambda) T_{\mathrm{ARP}}(\lambda) Q(\lambda) \lambda \mathrm{d} \lambda}{\int_{0}^{+\infty} \varphi(\lambda) M^{n}(\lambda) F_{x, y}(\lambda) T_{\mathrm{ARP}}(\lambda) Q(\lambda) \lambda \mathrm{d} \lambda} .
\end{aligned}
$$

Therefore, we can convert the count rate into the correct physical units in each passband $(x, y)$ because the abscal factor compensates for every variation in the optics efficiencies:

$\phi_{x, y}=\frac{K_{x, y \text { expected }}}{A_{x, y \text { theoretic }}} \cong \frac{K_{x, y \text { observed }}}{A_{x, y \text { real }}}$

deduced from Eq. (2).

In contrast, where differences between actual and laboratory measured profiles are asymmetric over the passbands, Eq. (7) is valid only in a first approximation, and the goodness of the approximation depends on the real shape of the passbands. In this case we could also find different absolute flux calibrations for every source observed. This may be the reason why we see a mismatch between Vega and $16 \mathrm{Cyg} \mathrm{A}+\mathrm{B}$ data in the UV range in Fig. 6. The steep UV behavior of the plot suggests that in this range, NAC and WAC passbands may have profiles with a shape different from that considered for the computation. These different shapes give an incorrect weight for computing the weighted arithmetic means that is at the base of the concept of Eq. (3).

These considerations are of fundamental importance for investigating possible aging or dust contamination of the optics during the last part of the mission around the comet, since we expect a wavelength-dependent variation of optical efficiencies in these cases. Therefore, we will have to continuously check this 
calibration: the computation of abscal factors may change with time, and the calibration will be good only because these factors compensate for efficiency variations. Moreover, an accurate computation of the correction profile $C(\lambda)$ deduced from $C_{x, y}$ must be implemented to improve the UV calibration.

One way to monitor the goodness of the approximation in Eq. (6) is to compute, for example, the flux calibration by means of different sources, with different spectra $\varphi(\lambda)$.

\subsection{List of UV calibrators}

We should therefore observe different stars with different spectral type to be able to check the consistency of the calibration independently of the star spectrum so that we can try to fix calibration issues after the awakening of OSIRIS. As we stated before, 16 Cyg A and B are solar analogs, with a very low S/N in the UV, which is the reason why at the moment only Vega is used at these wavelengths. We need stars different from Vega (A0V) and hotter than a solar analog $(\mathrm{G} 2 \mathrm{~V})$.

In Table 6 we present a list of stars in the BruzualPersson-Gunn-Stryker (BPGS) atlas with spectral type earlier than A3. These stars are cross-correlated with the TD1 catalog (Thompson et al. 1978) and the corresponding magnitudes are also provided (Hayes \& Latham 1975).

The BPGS atlas consists of 175 spectra of stars covering a complete range of spectral types and luminosity classes. Each individual spectrum covers the [229-25 600] A wavelength region. The fluxes were arbitrarily normalized to a visual magnitude of zero. More details about the spectra can be found in Gunn \& Stryker (1983).

\section{Reflectance calibration}

If we are observing a small body in the solar system, at the first approximation and at least in the visible range, we detect that the solar spectrum is diffused from the body surface. Considering OSIRIS data, we can remove the solar contribution on each image by computing the solar flux that falls inside each filter passband to obtain the spectrophotometric reflectance of the observed object inside each passband. We are able, therefore, to investigate its optical properties and give hints on its surface mineralogical composition.

For example, we wish to scale the image count rate so that if the target is observed at zero phase angle, it will give the value of the geometric albedo on the given passband. For that purpose, we recall some photometric definitions.

The geometric albedo is the ratio of a body brightness at zero phase angle to the brightness of a perfectly diffusing (Lambertian) disk with the same position and apparent size as the body. A Lambertian surface follows the cosine emission law (Lambert 1760): the intensity seen by an observer from an ideal diffusing surface is proportional to the cosine of the angle $\theta$ between the observer's line of sight and the normal to the surface, with peak luminous intensity $\left(I_{\text {peak }}\right)$ in the normal direction. The incident solar flux is equal to the total diffused flux that can be computed from the peak intensity by integrating the cosine law on the semi-sphere defined by the normal direction, obtaining $F_{\text {tot }}=\pi I_{\text {peak }}$.

The geometric albedo in a $(x, y)$ given passband is expressed by

$p_{x, y}=\frac{I_{x, y}}{\varphi_{\odot x, y}(r) / \pi}$, where $I_{x, y}$ is the body intensity at zero phase angle, $\varphi_{\odot x, y}(r)$ is the incident solar spectral irradiance at the body distance $r$ from the Sun, and the factor $\pi$ comes from the integral on the semi-sphere of the Lambert cosine law.

We can accordingly define the reflectance $R$ of a solid object illuminated by the Sun at a given phase angle $\alpha$ as

$R_{x, y}(\alpha)=\frac{I_{x, y}(\alpha)}{\varphi_{\odot x, y}(r) / \pi}$,

where $I_{x, y}(\alpha)$ is the intensity emitted by the body in the $(x, y)$ band at the phase angle $\alpha$ and $\varphi_{\odot x, y}(r)$ is the peak of the solar flux diffused by the Lambertian disk with the same cross-section of the object at the distance $r$ from the Sun in the same band.

From the solar spectrum at 1 AU (Burlov-Vasiljev et al. $1995,1998)$ we can compute the expected solar count rate at $1 \mathrm{AU}$, according to Eq. (1):

$K_{x, y \odot}=\int_{0}^{+\infty} \varphi_{\odot}(\lambda) M^{n}(\lambda) F_{x, y}(\lambda) T_{\mathrm{ARP}}(\lambda) Q(\lambda) \frac{\lambda}{h c} \frac{\mathrm{AP}}{\mathrm{IG}} \mathrm{d} \lambda$,

expressed in $\mathrm{DN} \mathrm{s}^{-1}$, where $\varphi_{\odot}(\lambda)$ is the solar spectral irradiance at $1 \mathrm{AU}$ expressed in $\mathrm{W} \mathrm{m}^{-2} \mathrm{~nm}^{-1}$. The solar spectral irradiance at $1 \mathrm{AU}$ inside a particular filter $(x, y)$ is expressed by the weighted mean:

$\varphi_{x, y_{\odot}}=\frac{\int_{0}^{+\infty} \varphi_{\odot}(\lambda) M^{n}(\lambda) F_{x, y}(\lambda) T_{\mathrm{ARP}}(\lambda) Q(\lambda) \lambda \mathrm{d} \lambda}{\int_{0}^{+\infty} M^{n}(\lambda) F_{x, y}(\lambda) T_{\mathrm{ARP}}(\lambda) Q(\lambda) \lambda \mathrm{d} \lambda}$

After dividing it by $\pi$ and scaling it to the distance $r$ of the target to the Sun expressed in astronomic units, we obtain the value $\varphi_{\odot}(r) / \pi$, expressed in $\mathrm{W} \mathrm{m}^{-2} \mathrm{~nm}^{-1} \mathrm{sr}^{-1}$, used to obtain the (geometric) reflectance within a given passband. Note that the reflectance is a adimensional number.

As already cited, we used the spectrum of the Sun measured by Burlov-Vasiljev et al. (1995, 1998). Solar analogs can be significantly different from the Sun, especially in the UV range, where the Sun can be variable by up to $20 \%$ (Colina et al. 1996). See Appendix B for more details.

In the following we use the expression

$\frac{\varphi_{x, y \odot}}{\pi}=\frac{\int_{0}^{+\infty} \varphi_{\odot}(\lambda) M^{n}(\lambda) F_{x, y}(\lambda) T_{\mathrm{ARP}}(\lambda) Q(\lambda) \lambda \mathrm{d} \lambda}{\pi \cdot \int_{0}^{+\infty} M^{n}(\lambda) F_{x, y}(\lambda) T_{\mathrm{ARP}}(\lambda) Q(\lambda) \lambda \mathrm{d} \lambda}(@ 1 \mathrm{AU})$,

or

$$
\frac{\varphi_{x, y \odot}}{\pi}=\frac{K_{x, y \odot}}{\pi \cdot \int_{0}^{+\infty} M^{n}(\lambda) F_{x, y}(\lambda) T_{\mathrm{ARP}}(\lambda) Q(\lambda) \frac{\lambda}{h c} \frac{\mathrm{AP}}{\mathrm{IG}} \mathrm{d} \lambda},
$$

to indicate the mean spectral irradiance of the Sun at a distance of $1 \mathrm{AU}$ in the wavelength range defined by the passband $(x, y)$. Since the star observed by OSIRIS cannot be directly the Sun, but is a solar analog, the counts on the CCD refer to this second star. We can thus infer the "solar" counts from the analog counts $\left(K_{x, y} \mathrm{SA}\right)$ by using

$K_{x, y \odot}=K_{x, y \mathrm{SA}} 10^{-0.4\left(V_{\odot}-V_{\mathrm{SA}}\right)}$,

so that the reflectance factor is

$\frac{\varphi_{x, y \odot}}{\pi}=\frac{K_{x, y \mathrm{SA}} 10^{-0.4\left(V_{\odot}-V_{\mathrm{SA}}\right)}}{\pi \cdot \int_{0}^{+\infty} M^{n}(\lambda) \cdot F_{x, y}(\lambda) T_{\mathrm{ARP}}(\lambda) Q(\lambda) \frac{\lambda}{h c} \frac{\mathrm{AP}}{\mathrm{IG}} \mathrm{d} \lambda}$, 
S. Magrin et al.: Pre-hibernation performances of the OSIRIS cameras onboard the Rosetta spacecraft

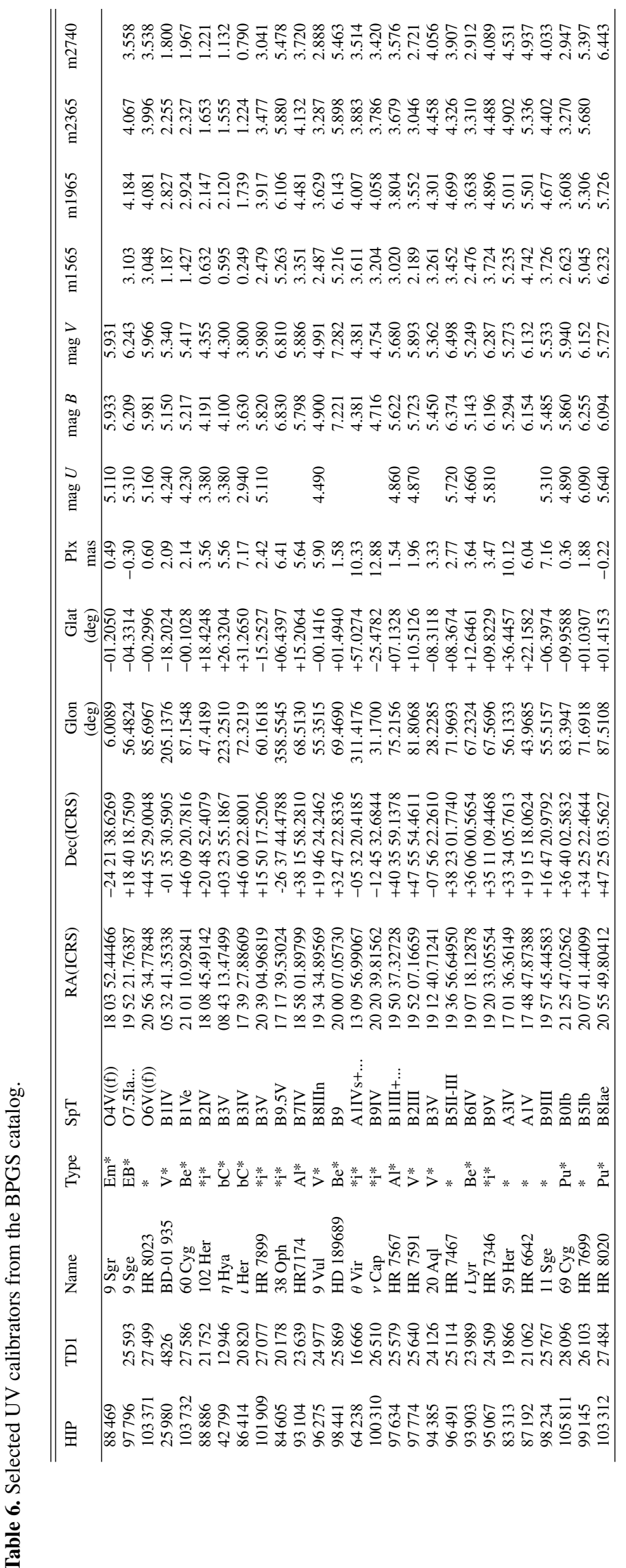

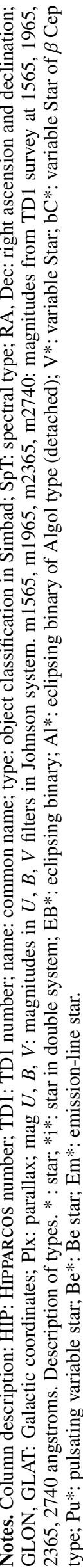


where the use of the $V$ magnitude, at each wavelength range, for the computation of the factor $10^{-0.4\left(V_{\odot}-V_{\mathrm{SA}}\right)}$ is justified since we assume the same spectral shape for the Sun and the solar analog. Some authors refer to the value of the solar flux as already divided by $\pi$. To avoid confusion, we always explicitly write $\varphi_{x, y \odot} / \pi$ as the solar intensity at $1 \mathrm{AU}$.

We can compute the reflectance directly from raw images (bias and flat-field corrected) by defining a composed abscal reflectance factor, multiplying the abscal factor defined in Eq. (3) and the new reflectance factor as expressed in Eq. (10).

Hence, the abscal reflectance factor defined is the value needed to convert the count rate into the reflectance of a body located at $1 \mathrm{AU}$ from the Sun:

$$
\begin{aligned}
A_{x, y} \cdot \frac{\varphi_{x, y}}{\pi}= & \frac{K_{x, y \text { calstar }} \cdot \text { pxsz }}{\pi} \\
& \times \frac{\int_{0}^{+\infty} \varphi_{\odot}(\lambda) M^{n}(\lambda) F_{x, y}(\lambda) T_{\mathrm{ARP}}(\lambda) Q(\lambda) \lambda \mathrm{d} \lambda}{\int_{0}^{+\infty} \varphi(\lambda)_{\text {calstar }} M^{n}(\lambda) F_{x, y}(\lambda) T_{\mathrm{ARP}}(\lambda) Q(\lambda) \lambda \mathrm{d} \lambda}
\end{aligned}
$$$$
\text { (@1 AU), }
$$

where the "calstar" source (calibration star, usually Vega) used for the absolute flux calibration is generally different from the "SA" source (solar analog, usually $16 \mathrm{Cyg} \mathrm{A}+\mathrm{B}$, or $16 \mathrm{Cyg} \mathrm{B}$ ) used to compute the solar value in Eq. (11). In Table 4 we report the abscal reflectance factor values computed by means of Eq. (12).

To derive the value needed for a body at a heliocentric distance $r$, we simply have to divide this factor by $r^{2}$ if $r$ is expressed in AU.

\subsection{Counts from the solar analog}

In the visible range the $\mathrm{S} / \mathrm{N}$ of $16 \mathrm{Cyg} \mathrm{B}$ for the WAC is better than 100 with $180 \mathrm{~s}$ exposure time even with narrowband filters. For the NAC, the $\mathrm{S} / \mathrm{N}$ is better than 200 with an exposure of a few seconds from about $480 \mathrm{~nm}$ to $1000 \mathrm{~nm}$. In this spectral range we can also use this solar analog as a flux calibration star. Equation (12) can thus be further simplified by using "SA" as "calstar", giving

$A_{x, y} \cdot \frac{\varphi_{x, y \odot}}{\pi}=\frac{K_{x, y} \mathrm{SA} \times \mathrm{pxsz} \times 10^{-0.4\left(V_{\odot}-V_{\mathrm{SA}}\right)}}{\pi} @ 1 \mathrm{AU}$,

since we used $\varphi_{\odot}=\varphi_{\mathrm{SA}} 10^{-0.4\left(V_{\odot}-V_{\mathrm{SA}}\right)}$.

This leads to smaller error bars because we simplified every instrumental passband; the passbands are the parameters carrying the largest uncertainties. Moreover, through this method we can exclude some problems that are due to the instrumental variation with time (age) and with some uncertainties in the optics efficiencies because they all affect the target and stellar images in the same way.

However, we have to remember that there may be some differences between the Sun and the solar analog, especially in the $\mathrm{UV}$, where the calibration with both $16 \mathrm{Cyg} \mathrm{A}+\mathrm{B}$ may lead to further approximations, and that this solar analog is quite faint in this wavelength range so that we have to work with a low $\mathrm{S} / \mathrm{N}$ in the $U V$ filters.

UV counts of 16 Cyg $A+B$ have large error bars because the spectrum is very weak in these wavelengths. In this case, the only way to find a reflectance value is to return to Eq. (12), computing the count rate from a star like Vega (which is much brighter than the Sun, especially in the UV). The division by the weighted Vega spectrum and the multiplication by the weighted
Sun spectrum corrects the difference caused by the two different spectral types.

The two methods are expected to give the same values. If the absolute calibration is reliable and if the abscal factors are the same regardless of the calibration star used, we can choose one of the two formulations to compute the best reflectance factor just according to the smallest error bars associated with the result.

Vega is a single star and has a much better $S / N$ than $16 \mathrm{Cyg}$ $\mathrm{A}$ or $\mathrm{B}$ in each filter. After checking that the calibration is consistent when computed using different calibrators, we found that the best star to use is Vega, both for the abscal factor computation and for the solar values computation.

This formulation was verified to be valid. Good matches, within error bars, between OSIRIS spectrophotometry and literature reflectance spectra can be found for Phobos (Pajola et al. 2012, 2013) and Lutetia (Magrin et al. 2012).

\subsection{Twin combinations}

When we have to calibrate NAC F84 images from NAC F24 baseline acquisition, we have to substitute Eq. (4) into Eq. (12), finding

$$
\begin{aligned}
A_{84} \cdot \frac{\varphi_{84 \odot}}{\pi}= & \frac{K_{24 \text { calstar }} \cdot \mathrm{pxsz}}{\pi} \\
& \times \frac{\int_{0}^{+\infty} \varphi_{\odot}(\lambda) \cdot M^{n}(\lambda) \cdot F_{84}(\lambda) T_{\mathrm{ARP}}(\lambda) Q(\lambda) \lambda \mathrm{d} \lambda}{\int_{0}^{+\infty} \varphi_{\text {calstar }}(\lambda) \cdot M^{n}(\lambda) \cdot F_{24}(\lambda) T_{\mathrm{ARP}}(\lambda) Q(\lambda) \lambda \mathrm{d} \lambda} \\
& @ 1 \mathrm{AU}
\end{aligned}
$$

This method was applied to complete the absolute reflectance calibration presented in Table 4 for filters without direct observation of the calibration stars.

\section{Conclusions}

We detected a good stability within $\pm 1.5 \%$ of the instrumental performances, without evidence of aging effects on the optics from 2007 to 2010. We found a good match between observed and expected data as deduced from on-ground laboratory measured optics and CCD efficiencies in the visible range. In the UV and IR wavelength range, however, we noted lower instrumental efficiencies than expected.

A reliable formulation for the flux calibration was proposed to be able to monitor this behavior from just after the exit from hibernation of the cameras and during the whole journey toward and around the comet, to study the possible dust contamination of the optics that would imply a wavelength-dependent variation of optical efficiencies. We also presented the formulation for the reflectance calibration. This formulation was verified to be valid, since there are good matches between OSIRIS spectrophotometry and literature reflectance spectra for Phobos (Pajola et al. 2012, 2013) and Lutetia (Magrin et al. 2012).

While the calibration compensates for instrumental efficiency variations in the visible and IR range, a deeper investigation of UV filters must be implemented. For this purpose, we finally suggested a list of other calibration sources. This will ensure a good calibration across the whole wavelength range during the entire last part of the mission.

Acknowledgements. OSIRIS was built by a consortium led by the Max-PlanckInstitut für Sonnensystemforschung, Katlenburg-Lindau, Germany, in collaboration with CISAS, University of Padova, Italy, the Laboratoire d'Astrophysique 
S. Magrin et al.: Pre-hibernation performances of the OSIRIS cameras onboard the Rosetta spacecraft

Table B.1. Properties of the Sun, 16 Cyg A, and 16 Cyg B according to Takeda (2005) and Takeda et al. (2005).

\begin{tabular}{rrlrcccccc}
\hline \hline Star & Sp. type & $T_{\text {eff }}$ & $V$ & $\pi$ & $M_{V}$ & B.C. & $\log \left(L / L_{\odot}\right)$ & $L / L_{\odot}$ & mass \\
\hline Sun & G2V & 5778 & -26.74 & & 4.83 & & & & 1 \\
HD 186408 16Cyg A & G1.5V & 5765.1 & 5.99 & 46.2 & 4.32 & -0.13 & +0.24 & 1.74 & 1.02 \\
HD 186427 16 Cyg B & G2.5V & 5795.4 & 6.25 & 46.7 & 4.60 & -0.12 & +0.10 & 1.26 & 1.03 \\
\hline
\end{tabular}

Notes. The HD number and the conventional stellar name for the three stars; the spectral type; the effective temperature derived from the spectroscopic approach reported in Takeda et al. (2005); the apparent $V$ magnitude and the trigonometric parallax $\pi$ in milliarcsecond, taken from the HIPPARCos Catalog (ESA 1997); the absolute $V$ magnitude, $M_{V}$, derived from the $\pi$ and $V$ values; the bolometric correction obtained from the results reported in Alonso et al. (1995); stellar luminosity $\log \left(L / L_{\odot}\right)$ and $L / L_{\odot}$ derived in Takeda et al. (2005) from $M_{V}$ and B.C.; stellar mass (in units of $M_{\odot}$ ) determined by $L / L_{\odot}$ and $T_{\text {eff }}$.

de Marseille, France, the Instituto de Astrofisica de Andalucia, CSIC, Granada, Spain, the Research and Scientific Support Department of the European Space Agency, Noordwijk, The Netherlands, the Instituto Nacional de Tecnica Aeroespacial, Madrid, Spain, the Universidad Politechnica de Madrid, Spain, the Department of Astronomy and Space Physics of Uppsala University, Sweden, and the Institut für Datentechnik und Kommunikationsnetze der Technischen Universität Braunschweig, Germany. The support of the national funding agencies of Germany (DLR), France (CNES), Italy (ASI), Spain (MEC), Sweden (SNSB), and the ESA Technical Directorate is gratefully acknowledged. This activity has been realized under the WAC/Rosetta Agenzia Spaziale Italiana (ASI) contract to the Istituto Nazionale di Astrofisica (INAF) I/024/12/0. We also thank the anonymous referee of the manuscript for providing constructive comments that helped to improve this paper.

\section{Appendix A: Units}

In the following we list and define the physical parameters and units we used.

- The irradiance is the power incident on a surface. It is expressed in the International System as $\mathrm{W} \mathrm{m}^{-2}$.

- The spectral irradiance is the power incident on a surface per unit wavelength, here expressed as $\mathrm{W} \mathrm{m}^{-2} \mathrm{~nm}^{-1}$.

- The radiance is the power emitted by a source per unit solid angle and unit area perpendicular to the emitting direction, expressed as $\mathrm{W} \mathrm{m}^{-2} \mathrm{sr}^{-1}$.

- The spectral radiance is the power per unit solid angle per unit area per unit wavelength, here expressed as $\mathrm{W} \mathrm{m} \mathrm{m}^{-2} \mathrm{~nm}^{-1} \mathrm{sr}^{-1}$.

The abscal factor presented in this paper is the factor needed to convert images from count rate into spectral radiance, so that the pixel-per-pixel signal associated with the target, observed in a particular passband, is directly comparable with a flux calibrated spectrum of the target in a wavelength interval around the central wavelength of the band.

\section{Appendix B: Description of the double star 16 Cyg A+B}

The two stars 16 Cyg A and B are slightly different from the Sun. In particular, 16 Cyg A is larger than 16 Cyg B, and both are larger than the Sun. From Takeda (2005) and Takeda et al. (2005) we can find the $\log L$ of both $16 \mathrm{Cyg} \mathrm{A}$ and $\mathrm{B}$, in terms of $L_{\odot}$, transformed from the absolute visual magnitude $M_{V}$ by means of the bolometric correction (B.C.) obtained by interpolating the results reported in Alonso et al. (1995). We summarize these results in Table B.1, where $T_{\text {eff }}$ is computed for all stars from a spectroscopic method using the equivalent width values of FeI and FeII lines. Both 16 Cyg A and B are very close to the Sun.
If placed at the same distance as the Sun, the flux of 16 Cyg $\mathrm{B}$ is about 1.26 times the solar flux, and the flux of the pair 16 Cyg $A+B$ is about three times the solar flux.

In summary: $16 \mathrm{Cyg} A$ and $B$ are slightly different from the Sun, but they seem to be similar enough to the Sun to allow us to assume the same spectral shape for the three stars, at least to the first order. Then, the simplest way to compute the scaling factor to convert the flux of $16 \mathrm{Cyg} \mathrm{A}+\mathrm{B}$ into the solar flux is to consider $V_{16 \mathrm{CygA}}=5.99, V_{16 \mathrm{CygB}}=6.25$, and $V_{\odot}=-26.74$ at $1 \mathrm{AU}$. The magnitude $\mathrm{V}$ of the pair is then

$V_{16 \text { Cyg A+B }}=-2.5 \cdot \log _{10} 10^{-0.4 \cdot V_{16 C y g A}}+10 .^{-0.4 \cdot V_{16 \mathrm{CygB}},}$

and the difference between the magnitude $V$ of the $16 \mathrm{Cyg}$ pair and the Sun leads to the relation

$\varphi_{16 \mathrm{Cyg} \mathrm{A}+\mathrm{B}}=\varphi_{\odot} \cdot 10^{-0.4 \cdot\left(V_{16 \mathrm{CygAB}}-V_{\odot}\right)}$

because we assume this factor to be a constant along the spectrum, since we assume the same spectral shape for the three stars.

To estimate the count rate of the Sun from the count rate of $16 \mathrm{Cyg} \mathrm{A}+\mathrm{B}$, the relation is

$K_{x, y \odot}=K_{x, y} 16 \mathrm{Cyg} \mathrm{A}+\mathrm{B} / 10^{-0.4 \times\left(V_{16 \mathrm{Cyg} \mathrm{AB}}-V_{\odot}\right)}$

at $1 \mathrm{AU}$, as we wrote in previous paragraphs.

\section{References}

Alonso, A., Arribas, S., \& Martinez-Roger, C. 1995, A\&A, 297, 197

Bohlin, R. C., \& Gilliland, R. L. 2004, AJ, 127, 3508

Burlov-Vasiljev, K. A., Gurtovenko, E. A., \& Matvejev, Y. B. 1995, Sol. Phys., 157,51

Burlov-Vasiljev, K. A., Matvejev, Y. B., \& Vasiljeva, I. E. 1998, Sol. Phys., 177, 25

Colina, L., Bohlin, R. C., \& Castelli, F. 1996, AJ, 112, 307

Dohlen, K., Jorda, L., Lamy, P., Toth, I., \& Origne, A. 2010, SPIE Conf. Ser., 7731

Gunn, J. E., \& Stryker, L. L. 1983, ApJS, 52, 121

Hayes, D. S., \& Latham, D. W. 1975, ApJ, 197, 593

Keller, H. U., Barbieri, C., Lamy, P., et al. 2007, Space Sci. Rev., 128, 433

Lambert, J. H. 1760, Photometria, sive, De mensura et gradibus luminis, colorum et umbrae (Augustae Vindelicorum: V.E. Klett)

Magrin, S., La Forgia, F., Pajola, M., et al. 2012, Planet. Space Sci., 66, 43

Pajola, M., Lazzarin, M., Bertini, I., et al. 2012, MNRAS, 427, 3230

Pajola, M., Lazzarin, M., Dalle Ore, C. M., et al. 2013, ApJ, 777, 127

Takeda, Y. 2005, PASJ, 57, 83

Takeda, Y., Ohkubo, M., Sato, B., Kambe, E., \& Sadakane, K. 2005, PASJ, 57, 27

Thomas, N., Keller, H. U., Arijs, E., et al. 1998, Adv. Space Res., 21, 1505

Thompson, G. I., Nandy, K., Jamar, C., et al. 1978, Catalogue of stellar ultraviolet fluxes A compilation of absolute stellar fluxes measured by the Sky Survey Telescope (S2/68) aboard the ESRO satellite TD-1 